\title{
Hipoventilação relacionada ao sono de origem central secundária à deficiência de biotinidase: relato de caso
}

\author{
Sleep-related hypoventilation due to partial biotinidase deficiency: a case report
}

Andressa Fernandes Silva ${ }^{1}(\mathbb{D})$, Carolina Vicentim de Moraes $^{1}\left(\mathbb{D}\right.$, Guilherme Andrade Pellissari ${ }^{1}(\mathbb{D})$, Zumira Aparecida Carneiro ${ }^{1}$ (D), Marcela Lopes de Almeida ${ }^{1,2}$ (D), Taciane Borsatto ${ }^{3,4}$ (D), Ida Vanessa Doederlein Schwartz ${ }^{3,4}$ (D), Charles Marques Lourenço ${ }^{1}$ (D)

\begin{abstract}
RESUMO
A hipoventilação relacionada ao sono de origem central resulta em hipercapnia relacionada ao sono na vigência de condições normais do sistema respiratório e excluindo-se outros fatores. Os pacientes portadores dessa patologia podem se apresentar assintomáticos ou com queixas de cefaleia matinal, déficit cognitivo e fadiga, além de eventos como a observação de respiração superficial. No presente relato, descreve-se o caso de uma paciente de três anos, com exame físico geral e neurológico normais, desenvolvimento neuropsicomotor adequado, apresentando irregularidades respiratórias e bradicardia durante o sono. Encaminhada para investigação de distúrbios respiratórios do sono, sendo diagnosticada com hipoventilação relacionada ao sono. Através do estudo genético, evidenciou-se a deficiência de biotinidase como a possível causa da sintomatologia, comprovada por dosagens enzimáticas e teste genético molecular. O tratamento medicamentoso foi iniciado precocemente, determinando resolução dos sintomas descritos. A importância do presente relato se encontra na apresentação da deficiência da biotinidase com quadro cardiorrespiratório isolado em criança neurologicamente normal, ademais trata-se de um caso em que a etiologia de Breath-Holding Spells foi a deficiência dessa enzima. Correspondência sugerida pela resolução da hipoventilação central após a introdução da biotina. Além disso, nesse caso, os sintomas Apparent Life-Threatening Events, que aterrorizam o observador e até o profissional, foram solucionados com tratamento simples, a ingesta oral de biotina. Esse relato de caso corrobora com a expansão das possibilidades de manifestações fenotípicas das formas tardias de deficiência de biotinidase, como o fenótipo da Síndrome da Hipoventilação Central.
\end{abstract}

Palavras Chaves: Deficiência de Biotinidase; Suspensão da Respiração; Hipoventilação.

\begin{abstract}
Idiopathic sleep-related hypoventilation occurs in individuals with hypercapnia during sleep in normal conditions of the respiratory system in the absence of other disorders. Patients with this condition may be asymptomatic or have complaints of morning headache, cognitive deficit and fatigue, and observation of shallow breathing. This report describes the case of a 3-year-old patient with normal physical and neurological exam, appropriate neuropsychomotor development, presenting breathing irregularities, and bradycardia during sleep. The patient was referred to an investigation for sleep respiratory disturbs and was diagnosed with hypoventilation related to sleep. The genetic study, done by enzymatic dosages and molecular genetic tests, showed the deficiency of biotinidase as a possible cause of symptomatology. The drug treatment was initiated early with the resolution of the symptoms. The present clinical report highlights the biotinidase deficiency with an isolated cardiorespiratory condition in a neurologically normal child, which also led to Breath-Holding Spells. This relation was suggested after central hypoventilation resolution following biotin introduction. Besides, Apparent Life-Threatening Events symptoms, which terrify the observer and even professionals, disappeared after the oral intake of biotin. Finally, this case report corroborates the expansion of possibilities for the phenotypic manifestations of late cases from biotinidase deficiency, as the SHC phenotype.
\end{abstract}

Keywords: Biotinidase Deficiency; Breath Holding; Hypoventilation

Centro Universitário Estácio de Ribeirão Preto, Ribeirão Preto (SP), Brasil.

Hospital das Clínicas da Faculdade de Medicina de Ribeirão Preto (HCFMRP), Ribeirão Preto (SP), Brasil.

Laboratory of Basic Research and Advanced Investigations in Neurosciences (BRAIN), Hospital de Clínicas de Porto Alegre (HCPA), Porto Alegre (RS), Brasil.

4 Universidade Federal do Rio Grande do Sul (UFRGS), Porto Alegre (RS), Brasil.

https://doi.org/10.11606/issn.2176-7262.rmrp.2021.166390

Medicina (Ribeirão Preto) 2021;54(1):e-166390 


\section{INTRODUÇÃO}

A hipoventilação relacionada ao sono de origem central resulta em hipercapnia relacionada ao sono na vigência de condições normais do sistema respiratório, incluindo vias aéreas, parênquima pulmonar, musculatura ventilatória e caixa torácica, e excluindo-se outros fatores como obesidade, outros distúrbios respiratórios do sono e uso de medicações depressoras do sistema nervoso central. Estima-se prevalência, na literatura, de 1 em cada 20.000 nascidos vivos na França ${ }^{1}$.

Os pacientes podem se apresentar assintomáticos ou com queixas de cefaleia matinal, déficit cognitivo e fadiga, além de eventos como a observação de respiração superficial ${ }^{2}$. A evolução de casos graves pode incluir hipertensão pulmonar, arritmias, insuficiências respiratória e cardíaca, policitemia secundária à hipoxemia e disfunção cognitiva².

As etiologias envolvem, principalmente, hipotireoidismo, e disfunções hipotalâmicas, e síndromes genéticas, como as provocadas por mutações no gene PHOX2B. Os casos considerados idiopáticos podem se relacionar a condições não elucidadas ${ }^{1}$.

Em crianças, o diagnóstico de hipoventilação relacionada ao sono é estabelecido quando a $\mathrm{PCO} 2$ se encontra maior que $50 \mathrm{mmHg}$ por mais que $25 \%$ do tempo total de sono ${ }^{3}$.

No presente relato, descreve-se o caso de uma paciente de três anos, com irregularidades respiratórias e bradicardia durante o sono, encaminhada para investigação de distúrbios respiratórios do sono, no caso apneias, com polissonografia. A criança foi diagnosticada com hipoventilação relacionada ao sono, cuja investigação evidenciou como causa do quadro clínico deficiência de biotinidase. Neste relato, os dados foram obtidos através da revisão do prontuário com a autorização do comitê de ética do hospital e do paciente, obtida por assinatura do termo de consentimento livre e esclarecido.

\section{RELATO DE CASO}

Paciente do sexo feminino, três anos, segunda filha de casal não consanguíneo, nasci- da de parto cesáreo, a termo (idade gestacional: 38 semanas), sem intercorrências perinatais.

Genitora observou desde os sete meses irregularidades respiratórias durante o sono, como respiração por vezes superficial ou periódica, associada à bradicardia. A criança apresentava antecedentes de refluxo gastresofágico e Apparent Life-Threatening Events (ALTE) desde os dois meses de idade, com episódios de pausas respiratórias em períodos de vigília, associados à cianose ou palidez e síncope, desencadeados por choro ou regurgitações.

O exame físico geral e o exame neurológico eram normais, com desenvolvimento neuropsicomotor adequado. A investigação complementar envolveu exames de eletrocardiograma, ecocardiograma transtorácico e eletroencefalograma, todos normais. Exame de Holter evidenciou irregularidade de intervalo R-R. Hemogramas seriados foram compatíveis com plaquetose (783 $\left.\times 10^{3} / \mathrm{uL}\right)$, com ferritina normal e sem causa aparente. Investigação inicial para erros inatos do metabolismo foi inconclusiva: houve apenas aumento da amônia sanguínea (107uG\%; valor de referência menor que $80 \mathrm{uG} \%$ ) e o lactato sérico apresentou-se elevado em duas ocasiões $(2,6 \mathrm{mmol} / \mathrm{L}$ e $4,8 \mathrm{mmol} / \mathrm{L}$; valor de referência de 0,5 a $2 \mathrm{mmol} / \mathrm{L})$. As gasometrias arteriais em vigília eram normais ou com alterações leves e inespecíficas (arterial: pH 7,38 / pO2 92,1 $\mathrm{mmHg} / \mathrm{pCO} 231,8 \mathrm{mmHg} / \mathrm{HCO} 318,3 \mathrm{mEq} / \mathrm{l} /$ BE -5,8 / SatO2 97,2 \% / Temperatura $37^{\circ} \mathrm{C}$; venosa $\mathrm{pH} 7,37$ / pO2 42,8 mmHg/ pCO2 $36,2 \mathrm{mmHg} / \mathrm{HCO} 320,6 \mathrm{mEq} / \mathrm{l} / \mathrm{BE}-4,1$ / Sat $0275,6 \%$ / Temperatura $37{ }^{\circ} \mathrm{C}$ ). Exame de neuroimagem foi a princípio evitado, em face do exame neurológico normal e do possível risco anestésico associado.

A polissonografia diagnóstica evidenciou tempo total de sono de 6,6 horas, eficiência do sono de $77,5 \%$, índice de microdespertares de $15,1 \%$, sono $\mathrm{N} 110,3 \%$, sono $\mathrm{N} 243,9 \%$, sono $\mathrm{N} 3$ $16,7 \%$, sono REM $29,1 \%$, Índice de Apneias Obstrutivas e Hipopneias (IAOH) de $1,7 / \mathrm{h}$ e Índice de Apneias Centrais (IAC) de $5,8 / \mathrm{h}$, saturação de oxigênio média de $96,8 \%$ e mínima de $86 \%$. Observou-se O IAC aumentado, todavia ainda dentro do limite superior para a faixa etária ${ }^{4}$. Ainda, foi documentado episódio de taquipneia 
após o último despertar, conforme uma das queixas clínicas. Neste estudo, não houve critérios quantitativos para diagnóstico de hipoventilação, segundo os critérios da American Academy of Sleep Medicine (AASM), uma vez que o tempo total de sono com ETCO2 $>50 \mathrm{mmHg}$ foi de apenas $5,2 \%{ }^{5}$. No entanto, observou-se que em $37,2 \%$ do tempo total de sono NREM, O ETCO2 esteve entre $45-49 \mathrm{mmHg}$, o que ocorreu em apenas $8,2 \%$ do tempo total de sono REM. Houve suspeita de hipoventilação incipiente de possível origem central, pela maior retenção de $\mathrm{CO} 2$ em sono NREM e pela ausência de sinais e sintomas de doença obstrutiva, a exemplo da Síndrome de Hipoventilação Alveolar Central Congênita.

A capnografia realizada como controle evolutivo demonstrou ETCO2 médio de $49,1 \mathrm{mmHg}$ e $60,4 \%$ do tempo total de sono com ETCO2 > $50 \mathrm{mmHg}$, o que desta vez confirmou diagnóstico de Hipoventilação, e documentou piora evolutiva ${ }^{3}$ (Tabela 1).

\section{Tabela 1}

Resultado de exame de capnografia antes do diagnóstico, demonstrando hipoventilação relacionada ao sono.

\begin{tabular}{lccccc}
\hline $\begin{array}{l}\text { ETCO2 } \\
(\mathrm{mmHg})\end{array}$ & $35-39$ & $40-44$ & $45-49$ & $50-59$ & $>60$ \\
$\begin{array}{l}\text { \% Tempo Total } \\
\text { de Registro }\end{array}$ & 1.9 & 15.2 & 17.5 & 60.4 & 0.0 \\
\hline
\end{tabular}

Fonte: Os autores (2018)

Como a investigação inicial para doenças metabólicas hereditárias foi inconclusiva, optou-se por realizar sequenciamento completo de exoma. Essa investigação evidenciou duas mutações em heterozigose composta no gene BTD, que codifica a enzima biotinidase, posteriormente confirmada pelo sequenciamento Sanger. Para verificar o efeito bioquímico dessas mutações, realizou-se teste enzimático na paciente, genitores e irmã assintomática (Tabela 2). Dosagens de biotinidase evidenciaram valores inferiores à normalidade $(1,4 \mathrm{nmol} / \mathrm{min} / \mathrm{mol}$; valor de referência de
4 a $10 \mathrm{nmol} / \mathrm{min} / \mathrm{mol}$ ), confirmada em laboratórios diferentes. A reposição de biotina $(20 \mathrm{mg} / \mathrm{dia})$ se associou à resolução dos sintomas descritos.

\section{Tabela 2}

Exame de análise molecular de DNA de sangue periférico da paciente e sua família para avaliação de mutações relacionadas à deficiência da biotinidase e as respectivas atividades dessa enzima.

\begin{tabular}{|c|c|c|c|}
\hline $\begin{array}{l}\text { Sujeito da } \\
\text { análise } \\
\text { molecular }\end{array}$ & $\begin{array}{c}\text { Variantes } \\
\text { encontradas }\end{array}$ & $\begin{array}{l}\text { Atividade da } \\
\text { Biotinidase }\end{array}$ & $\begin{array}{l}\text { Valor de } \\
\text { Referência }\end{array}$ \\
\hline Probando & $\begin{array}{l}\text { c.98_104de- } \\
\text { I7ins3 (p.C- } \\
\text { 33Ffs*36) / } \\
\text { c. } 1330 G>C \\
(\text { p.D444H) }\end{array}$ & $\begin{array}{c}1,4 \mathrm{nmol} / \mathrm{min} / \\
\mathrm{mol}\end{array}$ & $\begin{array}{c}4 \mathrm{a} 10 \\
\mathrm{nmol} / \mathrm{min} / \\
\mathrm{mol}\end{array}$ \\
\hline Pai & $\begin{array}{l}\text { c.98_104de- } \\
\text { I7ins3 (p.C- } \\
\text { 33Ffs*36) em } \\
\text { heterozigose }\end{array}$ & $\begin{array}{c}3,6 \mathrm{nmol} / \mathrm{min} / \\
\mathrm{mol}\end{array}$ & $\begin{array}{c}4 \text { a } 10 \\
\mathrm{nmol} / \mathrm{min} / \\
\mathrm{mol}\end{array}$ \\
\hline Mãe & $\begin{array}{c}1330 \mathrm{G}>\mathrm{C}(\mathrm{p} . \\
\mathrm{D} 444 \mathrm{H}) \mathrm{em} \\
\text { heterozigose }\end{array}$ & $\begin{array}{c}3,6 \mathrm{nmol} / \mathrm{min} / \\
\mathrm{mol}\end{array}$ & $\begin{array}{c}4 \text { a } 10 \\
\mathrm{nmol} / \mathrm{min} / \\
\mathrm{mol}\end{array}$ \\
\hline Irmã & $\begin{array}{c}1330 \mathrm{G}>\mathrm{C}(\mathrm{p} . \\
\mathrm{D} 444 \mathrm{H}) \mathrm{em} \\
\text { heterozigose }\end{array}$ & $\begin{array}{c}4,6 \mathrm{nmol} / \mathrm{min} / \\
\mathrm{mol}\end{array}$ & $\begin{array}{c}4 \text { a } 10 \\
\mathrm{nmol} / \mathrm{min} / \\
\mathrm{mol}\end{array}$ \\
\hline
\end{tabular}

Fonte: Os autores (2018)

A polissonografia de controle, após 6 meses de início da reposição de biotina, evidenciou resolução da hipoventilação alveolar (Tabela 3).

\section{Tabela 3}

Resultado de exame de capnografia após início da terapêutica, demonstrando resolução da hipoventilação relacionada ao sono.

\begin{tabular}{lccccc}
\hline $\begin{array}{l}\text { ETCO2 } \\
(\mathrm{mmHg})\end{array}$ & $35-39$ & $40-44$ & $45-49$ & $50-59$ & $>60$ \\
$\begin{array}{l}\text { \% Tempo Total } \\
\text { de Registro }\end{array}$ & 6.7 & 88.1 & 1.8 & 0.0 & 0.0 \\
\hline
\end{tabular}

Fonte: Os autores (2018) 


\section{DISCUSSÃO}

A Síndrome da Hipoventilação Central (SHC) é uma desordem não progressiva que afeta principalmente o controle neuromuscular da respiração durante o sono. É de ocorrência rara, entretanto deve ser uma hipótese diagnóstica em indivíduos com hipoxemia e hipoventilação nos primeiros meses de vida ${ }^{6}$. Devemos suspeitar dessa patologia em situações de sintomas ALTE (apneia, alterações da coloração da pele e do tônus muscular); manifestações respiratórias durante o sono; sintomas convulsivos inexplicáveis; reação respiratória grave após administração de medicação de sedativos ou antiepilépticos; atraso do desenvolvimento neuropsicomotor com história prévia de manifestações de cianose ou palidez importantes; ausência de resposta em situações de hipercapnia ou hipoxemia?. A paciente relatada apresentava parte desses sintomas, sinais claros de hipoventilação durante o sono e apneias em vigília.

O diagnóstico da SHC depende da investigação e, consequente exclusão, de outras causas de hipoventilação. Como malformações do tronco cerebral e anomalias estruturais do SNC, doenças neuromusculares e cardiovasculares, hipotireoidismo severo, paralisia do diafragma e anormalidades ventilatórias ${ }^{8}$. A SHC pode ser causada por doenças de cunho genético, como a denominada síndrome de Ondine, ocasionada por mutações do gene PHOX2B (paired-like homeobox gene), localizado no cromossomo $4 \mathrm{p} 12$, que codifica fatores de transcrição responsáveis pela regulação do desenvolvimento do sistema nervoso autôno$\mathrm{mo}^{1}$. No relato exposto, a hipoventilação alveolar relacionada ao sono desencadeou no diagnóstico de deficiência de biotinidase, associação rara até o momento na literatura. Este caso, de deficiência de biotinidase, comprovado por dosagens enzimáticas e teste genético molecular, propõe que após a administração de biotina, houve resolução completa da hipoventilação nos padrões capnográficos durante o sono, sugerindo que causas de apneia nestes indivíduos podem simular quadros de hipoventilação central.

A deficiência da biotinidase é uma enfermidade genética que afeta diretamente a ação da enzima biotinidase na absorção e metabolismo da biotina a partir de fontes alimentares. É o resulta- do de mutações autossômicas recessivas no gene BTD, localizado no cromossomo $3 \mathrm{p} 25^{9}$. Com a falha ou diminuição da atividade da biotinidase, ocorre alteração no ciclo da biotinidase levando ao acúmulo de metabólitos tóxicos no organismo, os quais resultarão em sinais e sintomas acometendo variados sistemas. Particularmente, destacam-se as alterações neurológicas, como letargia, hipotonia, convulsões, ataxia, atraso no desenvolvimento e alterações cutâneas. A deficiência da biotinidase pode ser total (menor que 10\% da atividade média normal da enzima), sendo essa mais evidente na infância, ou parcial ( $10 \%$ a $30 \%$ da atividade média normal), podendo ser manifestada tardiamente ${ }^{10}$.

A deficiência de biotinidase na forma tardia manifesta-se com paraparesia e tetraparesia, fraqueza nos membros e problemas de visão ${ }^{11}$. No caso descrito, a paciente não apresenta os sintomas previamente citados que podem contribuir para a hipótese clínica da deficiência de biotinidase de início tardio. Tal fato evidencia que suas manifestações foram diferentes em relação até mesmo da condição atípica da doença, comumente descrita na literatura, pois seu quadro clínico era de Breath-Holding Spells.

O fenômeno de Breath-Holding Spells pode ser definido como apneias episódicas em crianças, seguidas de palidez ou cianose e, possivelmente, associadas à perda de consciência e alteração do tônus, desencadeados por choros devido à dor, medo ou irritação ${ }^{12}$. Os eventos duram, em média, menos que um minuto e se assemelham a crises epilépticas, porém não se observam alterações no eletroencefalograma. Portanto, os episódios não causam prejuízos neurológicos às crianças. São mais comuns em crianças dos seis aos dezoito meses de vida e geralmente cessam aos cinco anos ${ }^{13}$. A paciente do presente relato possuía um quadro de Breath-Holding Spells que era desencadeado por choro ou regurgitações, após início de administração da biotina não apresentou mais o evento.

Além do método bioquímico, o diagnóstico pode ser feito através de um estudo do material genético, para deteç̧ões de mutações no gene biotinidase $^{14}$. 0 tratamento deve ser iniciado precocemente, a fim de minimizar e sanar a sintomática que a patologia acarreta. Consiste em reposição por via oral da biotina em doses de $5 \mathrm{mg} / \mathrm{dia}$ até $20 \mathrm{mg} / \mathrm{dia}^{15}$. 
A importância do presente relato se encontra na apresentação da deficiência da biotinidase com quadro cardiorrespiratório isolado em criança neurologicamente normal, ademais trata-se de um caso em que a etiologia de Breath-Holding Spells foi a deficiência dessa enzima. Ambos quadros possíveis, entretanto, na prática, muitas vezes não correlacionados devido a essa atípica manifestação. Essa correspondência é sugerida pela resolução da hipoventilação central após a introdução da biotina.

Além disso, nesse caso, os sintomas ALTE, que aterrorizam o observador e até o profissional, foram solucionados com tratamento simples, a ingesta oral de biotina. Em função disso, a deficiência de biotinidase pode ser considerada no diagnóstico diferencial de ALTE, Breath-Holding Spells e distúrbios ventilatórios do sono em crianças, incluindo casos "idiopáticos" de hipoventilação alveolar relacionada ao sono de origem central. Esse relato de caso corrobora com a expansão das possibilidades de manifestações fenotípicas das formas tardias de deficiência de biotinidase, como o fenótipo da SHC.

\section{REFERÊNCIAS}

1. Síndrome de hipoventilação central congênita associada à doença de Hirschsprung: relato de caso e revisão de literatura [Internet]. [citado 10 de maio de 2018]. Disponível em: http://www.scielo.br/scielo.php?script=sci_arttext\&pid $=$ S0103-05822016000300374\&Ing $=p t \& t \mid n g=p t$

2. Atualização Terapêutica de Felício Cintra do Prado, Jairo de Almeida Ramos ... - Emilia Inoue Sato, Arnaldo Lopes Colombo, Durval Rosa Borges, Luiz Roberto Ramos, Lydia Masako Ferreira, Ruth Guinsburg - Google Livros [Internet]. [citado 21 de maio de 2018]. Disponível em: https://books.google. com.br/books?id=HWU9DwAAQBAJ\&pg =PA206\& $\mathrm{Ipg}=\mathrm{PA} 206 \& \mathrm{dq}=\mathrm{A}+\mathrm{evolu} \% \mathrm{C} 3 \% \mathrm{~A} 7 \% \mathrm{C} 3 \% \mathrm{~A} 30+\mathrm{de}+$ casos + graves + pode+incluir + hipertens $\%$ C $3 \%$ A $30+$ arterial+pulmonar, + arritmias, +insufici $\%$ C $3 \%$ AAncias+respirat\%C3\%B3ria+e+card\%C3\%ADaca, + policitemia + secund \% C 3\%A 1 ria + \% C $3 \%$ A $0+$ hipoxemia+e+disfun $\%$ C3\%A7\%C3\%A3o+cognitiva.\& source $=$ bl\&ots $=$ tZc $\mathrm{JHa} 1 \mathrm{SAP} \& \mathrm{sig}=$ Rakpk0sAoMr2HKsdEvquzR7oT8w\&hl =pt-BR\&sa $=X \&$ ved $=2$ ahUKEwiakNa33ZbbAhUKCpAKHUt4CmIQ6AEwAHoECAEQLw \# $v=$ onepage $\& q=A \% 20$ evolu $\%$ C $3 \% A 7 \%$ C $3 \%$ A $30 \%$ 20 de $\% 20$ casos $\% 20$ graves $\% 20$ pode $\% 20$ incluir $\%$ 20 hipertens $\%$ C3 $\%$ A30\%20arterial\%20pulmonar $\%$ 2C\%20arritmias $\% 2$ C $\% 20$ insufici $\%$ C $3 \%$ AAncias $\%$ 20respirat\%C3\%B3ria\%20e\%20card\%C3\%ADaca\% 2C\%20policitemia \% 20secund \%C3\%A1ria\% $20 \%$ C3\%A0\%20hipoxemia\%20e\%20disfun \%C3\%A7\% C3\%A30\%20cognitiva. \&f $=$ false
3. International Classification of Sleep Disorders-Third Edition - CHEST [Internet]. [citado 14 de maio de 2018]. Disponível em: https://journal.chestnet.org/article/ S0012-3692(15)52407-0/fulltext

4. Scholle S, Wiater A, Scholle HC. Normative values of polysomnographic parameters in childhood and adolescence: cardiorespiratory parameters. Sleep Med. 2011 Dec;12(10):988-96. [Internet]. [citado 24 de setembro de 2020]. Disponível em: https://pubmed.ncbi.nlm.nih.gov/ 22036104/

5. Berry RB, Brooks R, Gamaldo CE, Harding SM, Lloyd RM, Marcus $C L$ and Vaughn BV The AASM manual for the scoring of sleep and associated events: rules, terminology and technical specifications. 7th ed. Westchester: American Academy of Sleep Medicine; 2016. [Internet]. [citado 24 de setembro de 2020]. Disponível em: https:// aasm.org/resources/pdf/scoring-manual-preface.pdf

6. Liess BD, Dost JS, Templer JW, Tobias JD. Congenital central alveolar hypoventilation syndrome (Ondine's curse) with survival into adulthood. Clin Pediatr (Phila). 2008 Nov;47(9):941-6. [Internet]. [citado 21 de maio de 2018]. Disponível em: http://journals.sagepub.com/doi/ abs $/ 10.1177 / 0009922808320701$ ?related-urls = yes $147 \% 2$ F9\%2F941 \&legid = spcpj\%3B $47 \% 2$ F9\% 2F941\&cited-by $=$ yes \&

7. Weese-Mayer DE, Berry-Kravis EM, Ceccherini I, Keens TG, Loghmanee DA, Trang $H$. Édition française de la Déclaration officielle de politique clinique de I'American Thoracic Society (ATS) sur le syndrome d'hypoventilation alvéolaire centrale congénitale. Bases génétiques, diagnostic et prise en charge. Rev Mal Respir. $1^{\circ}$ de outubro de 2013;30(8):706-33.

8. Bachetti T, Matera I, Borghini S, Di Duca M, Ravazzolo R, Ceccherini I. Distinct pathogenetic mechanisms for PHO$\mathrm{X} 2 \mathrm{~B}$ associated polyalanine expansions and frameshift mutations in congenital central hypoventilation syndrome. Hum Mol Genet. 2005 Jul 1;14(13):1815-24. [citado 12 de maio de 2018]. Disponível em: https://www. researchgate.net/publication/7853715_Distinct_ pathogenic_mechanisms_for_PHOX2B_associated_ polyalanine_expansions_and_frameshift_mutations_in_ congenital_central_hypoventilation_syndrome

9. Nelson Tratado de Pediatria - Robert Kliegman, Bonita M.D. Stanton, Joseph St. Geme, Nina F Schor - Google Livros [Internet]. [citado 21 de maio de 2018]. Disponível em: https://books.google.com.br/books?id=iIE5DwAAQBAJ\& $\mathrm{pg}=\mathrm{PT} 2135 \& \mathrm{lpg}=\mathrm{PT} 2135 \& \mathrm{dq}=\% \mathrm{C} 3 \% 89+\mathrm{o}++$ resultado + de+muta\%C3\%A7\%C3\%B5es+autoss $\%$ C3\%B4micas+ recessivas+no+gene+BTD,+localizado+no+cromossomo+ 3 p25\&source $=$ bl\&ots $=1$ p6lxai 5 UH\&sig $=x 40 B a 7 \mid R h u R-$ ubHH8K4kPanh380\&hl =pt-BR\&sa $=$ X\&ved $=2$ ahUKEwjx5YCCIZfbAhXEIZAKHZhjDFAQ6AEwAnoECAEQRA\#v= onepage $\& q=\%$ C $3 \% 89 \% 200 \% 20 \% 20$ resultado $\% 20 \mathrm{de} \%$ 20muta\%C3\%A7\%C3\%B5es\%20autoss\%C3\%B4micas\% 20 recessivas $\% 20$ no $\% 20$ gene $\% 20$ BTD \% 2 C \% 20localizado $\% 20$ no $\% 20$ cromossomo $\% 203$ p $25 \&$ f =false

10. Wolf $B$. Clinical issues and frequent questions about biotinidase deficiency. Mol Genet Metab. 2010 May;100(1):6-13. [citado 10 de maio de 2018]. Disponível em: https://www.sciencedirect.com/science/article/ pii/S1096719210000041 
11. Wolf B, Pomponio RJ, Norrgard KJ, Lott IT, Baumgartner ER, Suormala T, et al. Delayed-onset profound biotinidase deficiency. J Pediatr. $1^{\circ}$ de fevereiro de $1998 ; 132(2): 362-5$.

12. Breath-Holding Spells [Internet]. Child Neurology Foundation. [citado 12 de maio de 2018]. Disponível em: http://www.childneurologyfoundation.org/disorders/ breath-holding-spells/

13. Ozcora GDK, Kumandaş $S$, Sağıroğlu A, Acer N, Doğanay $\mathrm{S}$, Yiğit $\mathrm{H}$, et al. Evaluating brainstem in breath-holding spells. Eur J Paediatr Neurol. $1^{\circ}$ de junho de 2017;21:e78.
14. C. Neto E, Schulte J, Rubim R, Lewis E, DeMari J, CastiIhos $C$, et al. Newborn screening for biotinidase deficiency in Brazil: biochemical and molecular characterizations. Braz J Med Biol Res. março de 2004;37(3):295-9.

15. Strovel ET, Cowan TM, Scott AI, Wolf B. Laboratory diagnosis of biotinidase deficiency, 2017 update: a technical standard and guideline of the American College of Medical Genetics and Genomics. Genet Med [Internet]. outubro de 2017 [citado 12 de maio de 2018];19(10). Disponível em: http://www.nature.com/ doifinder/10.1038/gim.2017.84

Autor correspondente:

Charles Marques Lourenço

charlesgenetica@gmail.com

Editor:

Prof. Dr Felipe Villela Gomes

Recebido em: 06/02/2020

Aprovado em: 16/11/2020

(c) Este é um artigo publicado em acesso aberto (Open Access) sob a licença Creative 